\title{
Exposure to combustion generated environmentally persistent free radicals enhances severity of influenza virus infection
}

Greg I Lee 1,2,3, Jordy Saravia, ${ }^{2,3}$, Dahui You ${ }^{2,3}$, Bishwas Shrestha ${ }^{2,3}$, Sridhar Jaligama ${ }^{2,3}$, Valerie Y Hebert ${ }^{4}$, Tammy R Dugas ${ }^{4}$ and Stephania A Cormier ${ }^{2,3^{*}}$

\begin{abstract}
Background: Exposures to elevated levels of particulate matter (PM) enhance severity of influenza virus infection in infants. The biological mechanism responsible for this phenomenon is unknown. The recent identification of environmentally persistent free radicals (EPFRs) associated with PM from a variety of combustion sources suggests its role in the enhancement of influenza disease severity.

Methods: Neonatal mice (< seven days of age) were exposed to DCB230 (combustion derived PM with a chemisorbed EPFR), DCB50 (non-EPFR PM sample), or air for 30 minutes/day for seven consecutive days. Four days post-exposure, neonates were infected with influenza intranasally at $1.25 \mathrm{TCI}_{50} /$ neonate. Neonates were assessed for morbidity (\% weight gain, peak pulmonary viral load, and viral clearance) and percent survival. Lungs were isolated and assessed for oxidative stress (8-isoprostanes and glutathione levels), adaptive immune response to influenza, and regulatory T cells (Tregs). The role of the EPFR was also assessed by use of transgenic mice expressing human superoxide dismutase 2.

Results: Neonates exposed to EPFRs had significantly enhanced morbidity and decreased survival following influenza infection. Increased oxidative stress was also observed in EPFR exposed neonates. This correlated with increased pulmonary Tregs and dampened protective $T$ cell responses to influenza infection. Reduction of EPFR-induced oxidative stress attenuated these effects.

Conclusions: Neonatal exposure to EPFR containing PM resulted in pulmonary oxidative stress and enhanced influenza disease severity. EPFR-induced oxidative stress resulted in increased presence of Tregs in the lungs and subsequent suppression of adaptive immune response to influenza.
\end{abstract}

Keywords: Particulate matter, EPFR, Influenza, Infant, Neonate, Air pollution, DCB230, Oxidative stress

\section{Background}

The associations between PM pollution exposure and adverse health outcomes are well documented. These adverse health effects include respiratory diseases such as chronic obstructive pulmonary disease and asthma [1-5]. Interestingly, associations between PM exposure and increased susceptibility to infectious respiratory diseases are also observed [6-8]. Due to the heterogeneous nature

\footnotetext{
* Correspondence: scormier@uthsc.edu

${ }^{2}$ Department of Pediatrics, University of Tennessee Health Sciences Center,

Memphis, Tennessee 38103, USA

${ }^{3}$ Children's Foundation Research Institute, Le Bonheur Children's Hospital,

Memphis, Tennessee, USA

Full list of author information is available at the end of the article
}

of PM pollution, there is ongoing interest in identifying the particularly hazardous components. Recent studies by our collaborators identify the presence of "environmentally persistent free radicals" (EPFRs) in PM samples collected from various cities in the United States [9]. These newly identified EPFRs are a prime candidate as a causative agent for adverse outcomes associated with PM exposures, since free radicals are known to cause negative biological health effects.

Although PM exposure affects people of all ages, its effects on young children and infants are particularly detrimental $[10,11]$. Similarly, respiratory viral infections, such as seasonal influenza, also maintain an age bias where 
the very young and elderly are particularly at risk of more severe disease [12]. Infants are a unique and particularly susceptible population to both viral respiratory illnesses and PM pollution due to their developing pulmonary and immunological systems; and their faster breathing rates compared to adults.

There is a paucity of studies that delve into the biological mechanisms responsible for PM exposure-enhanced respiratory viral illness. Our prior studies demonstrate that exposure of neonatal mice (i.e., mouse age when pulmonary and immune system mimics a human infant $[13,14])$ to combustion derived PM induces both airway epithelial damage and barrier dysfunction and results in a temporary immunosuppressive pulmonary microenvironment $[15,16]$. The epithelial damage and/or the suppressed pulmonary immune microenvironment could be responsible for epidemiological data demonstrating enhanced morbidity following respiratory viral infection following exposure to elevated levels of PM pollution.

We hypothesized that EPFRs in the combustion derived PM are responsible for enhanced influenza morbidity and decreased survival observed in infants. We are studying the EPFR of dichlorobenzene that is formed by reaction with $\mathrm{Cu}(\mathrm{II}) \mathrm{O}$ containing fly-ash at $230^{\circ} \mathrm{C}$ (referred to as DCB230) $[17,18]$. To address our hypothesis, we exposed neonatal mice to EPFR containing PM (DCB230), nonEPFR containing PM (DCB50), or DCB230 in the presence of elevated antioxidants and subsequently infected them with influenza.

\section{Results}

Acute inhalation exposure to EPFRs induces pulmonary oxidative stress in neonates

In a prior study, we observed oxidative stress in the lungs after seven days of exposure to DCB230 in neonatal rats [18] and bronchial epithelial cells [16]. To determine if oxidative stress was present earlier (within five days of exposure), three day old mice were exposed to air (Air), DCB50 (D50), or DCB230 (D230) (Figure 1A). After five days of exposure, we isolated the lungs and analyzed the lung homogenates for indicators of oxidative stress including 8-isoprostanes (8-IP) and glutathione/oxidized glutathione ratio (GSH/GSSG). Neonatal mice exposed to DCB230 had significantly higher levels of 8-IP compared to all other groups (Figure 1B). In addition, DCB230 exposed neonates had a significantly lower GSH/GSSG ratio compared to Air (Figure 1C). The GSH/GSSG ratio was not different between DCB230 and DCB50 exposed neonates. To help clarify the role of the EPFR in the induction of pulmonary oxidative stress, we also exposed neonatal mice that expressed human mitochondrial superoxide dismutase $\left(\mathrm{hSOD}^{+}\right)$to D230 $\left(\mathrm{D} 230^{(\mathrm{hSOD}+)}\right)$. The additional antioxidant capacity was sufficient to significantly reduce the amount of 8-IP produced in the lungs during acute

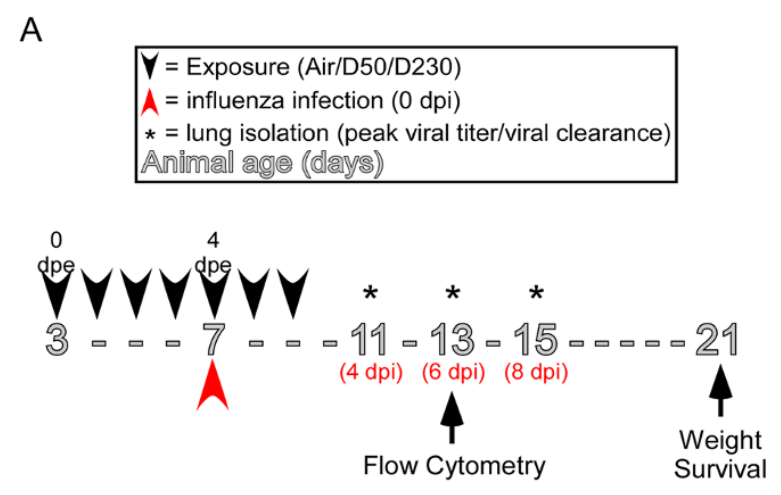

B

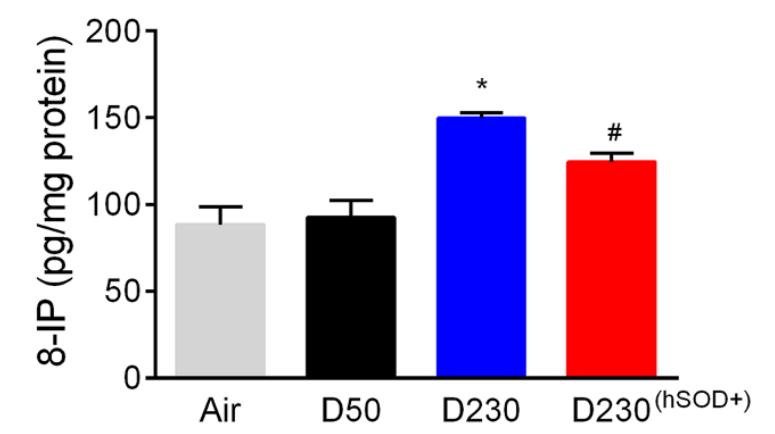

C

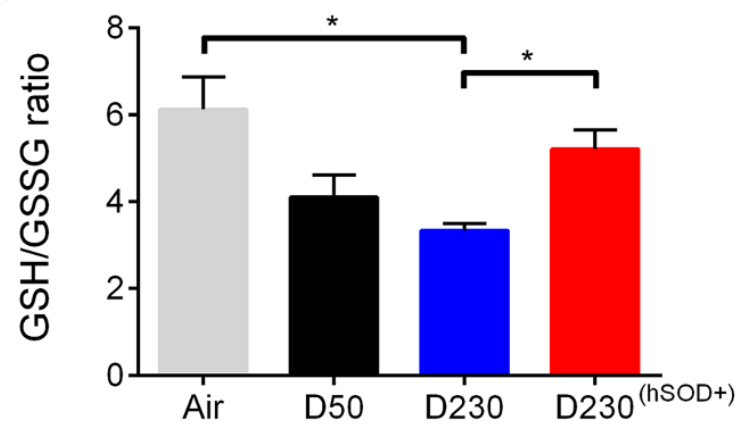

Figure 1 Effect of EPFR exposure on oxidative stress burden in neonates. A) Neonatal exposure and influenza infection model. Three day old mice were exposed to $200 \mu \mathrm{g} / \mathrm{m}^{3}$ of DCB230, DCB50, or air for seven consecutive days (0-7 dpe) for $30 \mathrm{~min} /$ day (black arrow head) and infected with influenza at 1.25 TCID50/neonate on the fourth dpe (red arrow head). Non-infected lungs were isolated at four dpe for analysis of oxidative stress and regulatory T cells (Tregs). Infected lungs were isolated for pulmonary viral load, flow cytometry, and pulmonary viral clearance on four, six, and eight days post-infection (dpi; asterisks). Pulmonary oxidative stress, determined by levels of (B) 8-isoprostance (IP) and (C) GSH/GSSG ratio in lungs of wild-type C57BL/6 after five days of exposure to air, DCB50 (D50), or DCB230 (D230) and in lungs of hSOD2 transgenic neonates exposed to DCB230 (D230 $\left.{ }^{(\mathrm{hSOD}+)}\right)$. Data plotted as mean \pm standard error of mean (SEM). ${ }^{*} p<0.05$ D230 vs Air, $\mathrm{D} 50$, and D230 ${ }^{(\mathrm{hSOD}+)} ; \# p<0.05 \mathrm{D} 230^{(\mathrm{hSOD+}+)}$ vs Air, D50, and D230; Brackets indicate $p<0.05$ D230 vs Air and D230 $0^{(\text {hSOD+)}}$; one-way analysis of variance (ANOVA) with Tukey's multiple comparisons test. N =4-10/group. 


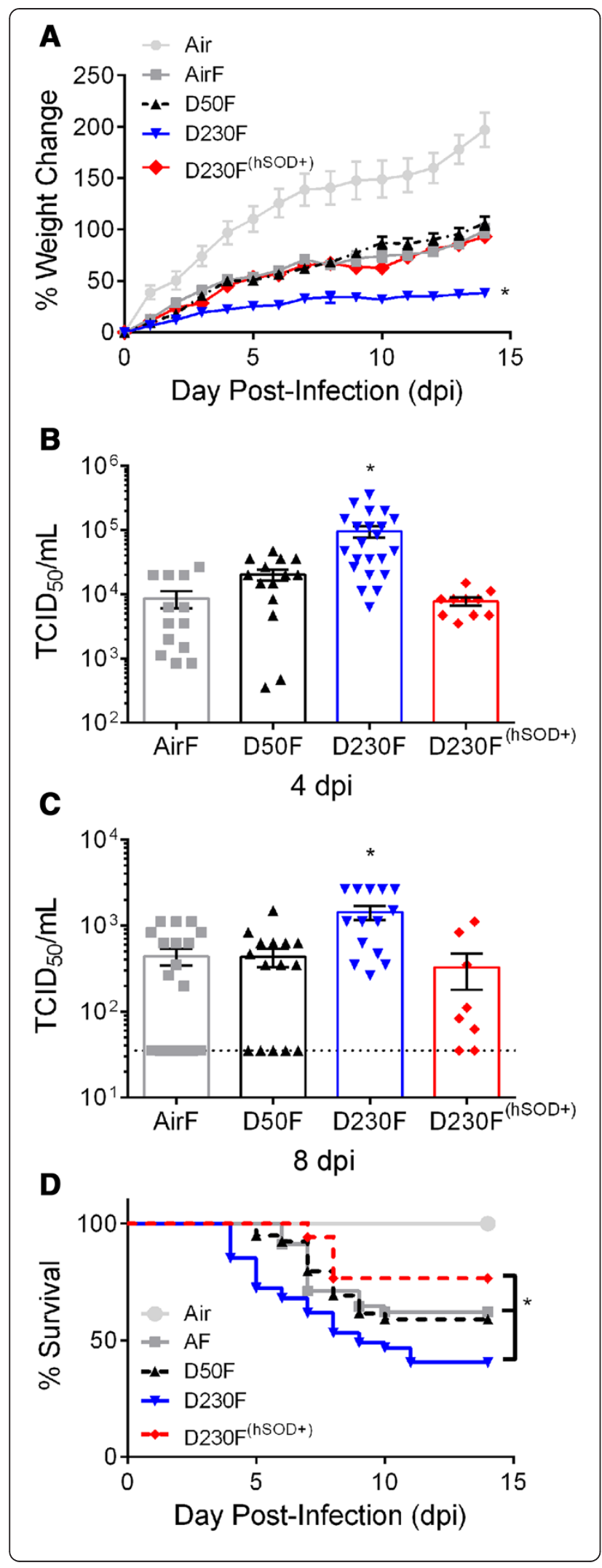

Figure 2 Effect of EPFR exposure on influenza morbidity and viral load in neonates. Neonatal mice were exposed to air, DCB50, or DCB230 and infected i.n. with influenza (AirF, D50F, D230F, D230F ${ }^{(\mathrm{hSOD+1})}$ ) at $1.25 \mathrm{TCID}_{50}$ /neonate or sham infected with DPBS (Air). (A) Average percent change in weight plotted against dpi. $N=16-35$ / group. (B) Peak pulmonary viral load assessed at four dpi. $N=10-22$ / group. (C) Pulmonary viral clearance assessed at eight dpi. Dotted line indicates limit of detection ( $35 \mathrm{TCID}_{50} / \mathrm{mL}$ ) of assay. (D) Effect of EPFR exposure on influenza infection associated drop in survival in neonates. Survival was recorded daily until 14 dpi. $N=8-18$ /group. Data plotted as mean \pm SEM. ${ }^{*} p<0.05$ D230F vs Air, AirF, D50F, and D230F ${ }^{(\mathrm{hSOD+})}$. (A) Multiple t tests with Holm-Sidak correction for multiple comparisons. (B and C) One-way ANOVA with Tukey's multiple comparisons test. (D) Gehan-Breslow Wilcoxon test with Bonferroni correction for multiple comparisons; ${ }^{*} p<0.014$ between D230F vs AirF and D230F ${ }^{(\mathrm{hSOD+})}$. N =17-47/group.

exposure to EPFRs and increase the ratio of GSH/GSSG. To ensure there were no inherent sub-strain differences between transgenic neonates and wild-type (WT) neonates, we compared oxidative stress responses in WT and hSOD2 neonates exposed to DCB230. No differences in normalized levels of 8-IP between WT (D230/Air: $1.65 \pm 0.054$ ) vs. hSOD $^{-}$(D230 ${ }^{\text {(hSOD-) }} /$ Air $^{\left(\mathrm{hSOD}^{-}\right)}: 1.61 \pm 0.18$; Additional file 1: Figure S1A) or normalized GSH/GSSG ratios (D230/Air: $0.54 \pm 0.03 ; \mathrm{D} 230^{(\mathrm{hSOD}-)} / \mathrm{Air}^{(\mathrm{hSOD}-)}: 0.55 \pm 0.07$; Additional file 1: Figure S1B) was identified.

\section{Oxidative stress is critical in the enhancement of influenza morbidity observed following exposure to EPFRs in neonates}

Epidemiological data demonstrate an increase in morbidity due to respiratory tract infections following high PM exposures [19-21]. Therefore, we assessed morbidity in the neonatal mice exposed to EPFRs. Mice were weighed daily to determine ability of influenza to induce weight loss and measured viral loads at the peak of viral replication and again four days later to assess the ability to control viral replication and to clear virus. Neonates were exposed to air or PM for seven consecutive days and sham (Air) or influenza (AirF, D50F, D230F) infected four days post-exposure (dpe; Figure 1A). Following infection, neonates exposed to DCB230 (D230F: $38.15 \pm 4.57 \%$; (Figure 2A) gained significantly less weight compared to all other groups (Air: 197.09 $\pm 16.74 \%$; AirF: $98.91 \pm$ 6.50\%; D50F: $106.23 \pm 6.54 \%$ ). Higher pulmonary viral loads were also observed in DCB230 exposed neonates (D230F: $95007 \pm 19657 \mathrm{TCID}_{50} / \mathrm{mL}$; Figure 2B) at four days post infection (dpi), which represents the peak viral load, compared to all other groups (AirF: $8626 \pm 2573$ $\mathrm{TCID}_{50} / \mathrm{mL} ; \mathrm{D} 50 \mathrm{~F}: 20198 \pm 3829 \mathrm{TCID}_{50} / \mathrm{mL}$ ). Furthermore, DCB230 exposed neonates continued to present with significantly higher pulmonary viral loads at eight dpi (D230F: $1432 \pm 265.5 \mathrm{TCID}_{50} / \mathrm{mL}$; Figure $2 \mathrm{C}$ ) compared to all other groups (AirF: $440.7 \pm 99.16 \mathrm{TCID}_{50} / \mathrm{mL}$; D50F: $\left.434.1 \pm 102.8 \mathrm{TCID}_{50} / \mathrm{mL}\right)$. Of note, the exacerbation in 


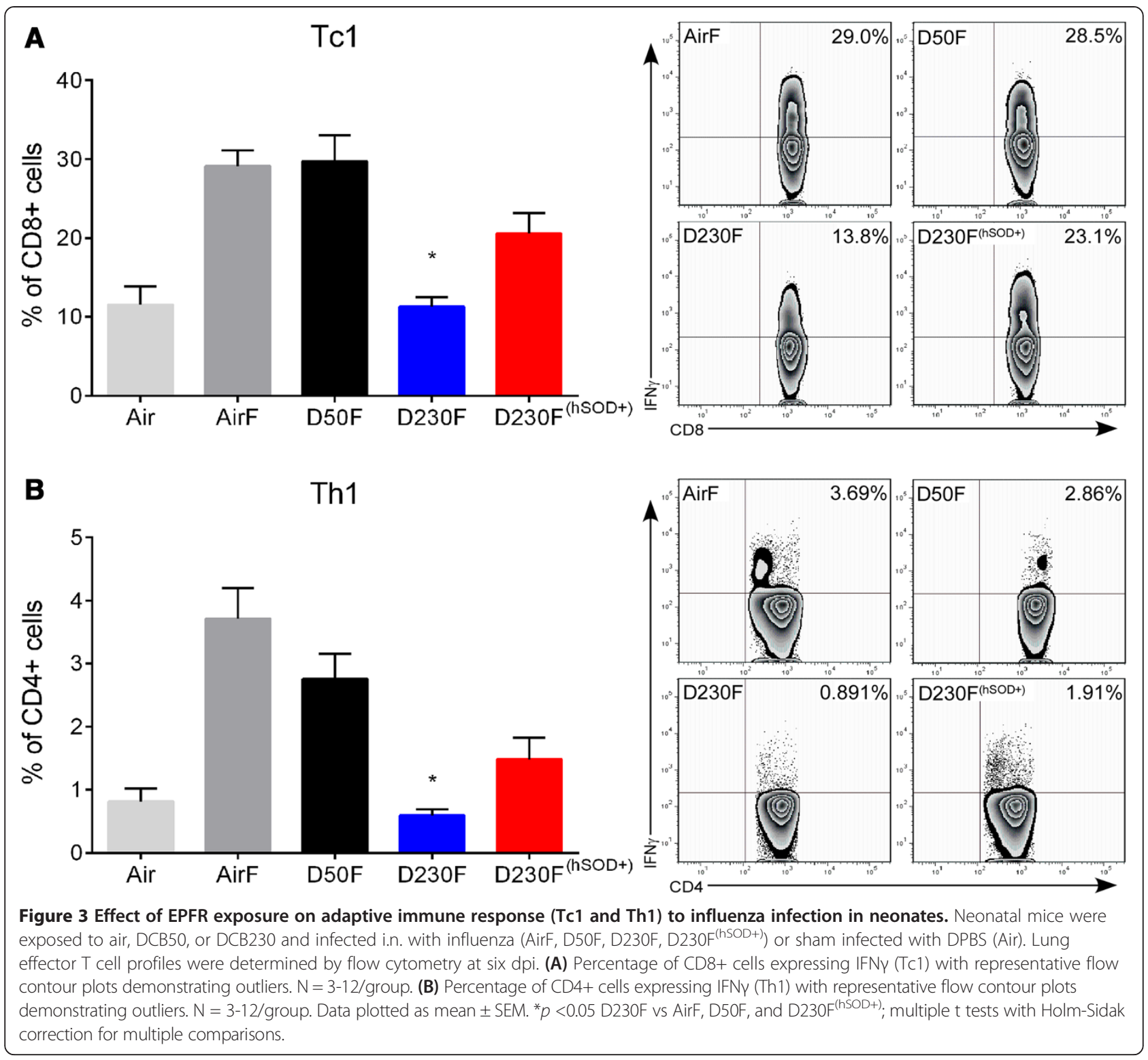

morbidity (decreased weight gain and increased viral load at four and eight dpi) due to EPFR exposure was reversed in $\mathrm{hSOD}^{+}$neonates (D230F ${ }^{(\mathrm{hSOD}+)}$ : $93.2 \pm 6.94 \%$; $7683 \pm$ $\left.1099 \mathrm{TCID}_{50} / \mathrm{mL} ; 327.8 \pm 147.5 \mathrm{TCID}_{50} / \mathrm{mL}\right)$. To ensure that these results were not due to sub-strain differences between WT and transgenic neonates, we also exposed WT and hSOD ${ }^{-}$neonates to air and DCB230 with subsequent intranasal (i.n.) influenza infection. There were no observed differences in pulmonary viral load at four dpi between WT (Additional file 2: Figure S2A) and hSOD' neonates exposed to DCB230 (AirF ${ }^{(\mathrm{hSOD}-)}$ : $7368 \pm 3156$ $\mathrm{TCID}_{50} / \mathrm{mL}$; $\left.230 \mathrm{~F}^{(\mathrm{hSOD}-)}: 146343 \pm 47621 \mathrm{TCID}_{50} / \mathrm{mL}\right)$ as well as at eight dpi $\left(\operatorname{AirF}^{(\mathrm{hSOD}-)}: 266.3 \pm 148.8 \mathrm{TCID}_{50} / \mathrm{mL}\right.$;

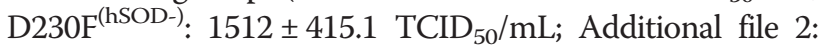
Figure S2B). In fact, DCB230 exposed hSOD ${ }^{-}$neonates were similar to their WT counterparts. Furthermore, there was no difference in pulmonary viral loads at four dpi between WT, hSOD ${ }^{+}$, or $\mathrm{hSOD}^{-}$mice (AirF: $482.3 \pm 62.34$ TCID $_{50} / \mathrm{mL} ; \operatorname{AirF}^{(\mathrm{hSOD}+)}: 1241 \pm 496.8$ $\mathrm{TCID}_{50} / \mathrm{mL} ; \quad \operatorname{AirF}^{(\mathrm{hSOD}+)}: 431.7 \pm 38.11 \mathrm{TCID}_{50} / \mathrm{mL}$; Additional file 3: Figure S3).

\section{EPFR exposure decreases survival following influenza infection}

To assess the effects of EPFR exposure on mortality following influenza infection in neonates, we recorded daily survival data to $14 \mathrm{dpi}$. We observed a significant drop in percent survival in neonates exposed to D230F compared to $\operatorname{AirF}(40.43 \%$ vs. $62 \%$; respectively; $p<0.014$ ) (Figure 2D). D50F exposed neonates had improved survival compared to D230F and was not statistically different from AirF. Again, the enhanced antioxidant capacity of $\mathrm{hSOD}^{+}$ 
neonates was able to significantly improve survival of mice exposed to DCB230 and infected with influenza (76.47\% vs. $40.43 \%$; respectively; $p<0.014$ ). Interestingly, D230F neonates consistently exhibited earlier mortality (four dpi) compared to all other groups.

\section{Protective immune responses against influenza are diminished following EPFR exposure}

To determine the effects of EPFR exposure on the adaptive immune response to influenza, lungs were isolated at six $\mathrm{dpi}$ and $\mathrm{T}$ cell profiles were assessed. $\mathrm{T}$ cells expressing IFN $\gamma$ are critical to control and clear influenza. The percent of CD8+ T cells expressing IFNy (Tc1) were significantly reduced in D230F neonates (Figure 3A) compared to AirF or
D50F. Furthermore, the percent of CD4+ T cells expressing IFN $\gamma$ (Th1) were significantly lower in D230F neonates (Figure 3B) compared to AirF and D50F. The increased antioxidant capacity afforded to $\mathrm{D} 230 \mathrm{~F}^{(\mathrm{hSOD}+)}$ neonates resulted in significant increases in the percentage of both the Tc1 and Th1 cells compared to D230F. To further confirm whether percent Tc1 and Th1 values show similar trends in terms of total number of lung Tc1 and Th1 cells, the percent data was transformed to and analyzed in terms of total lung Tc1 and Th1 cells. Similar to percent data, the total number of both Tc1 and Th1 cells were significantly reduced in D230F neonates compared to AF or D50F and increased antioxidant capacity in $\mathrm{D}_{230 \mathrm{~F}^{(\mathrm{hSOD}+)}}$ neonates resulted in a significant increase in total lung Tc1

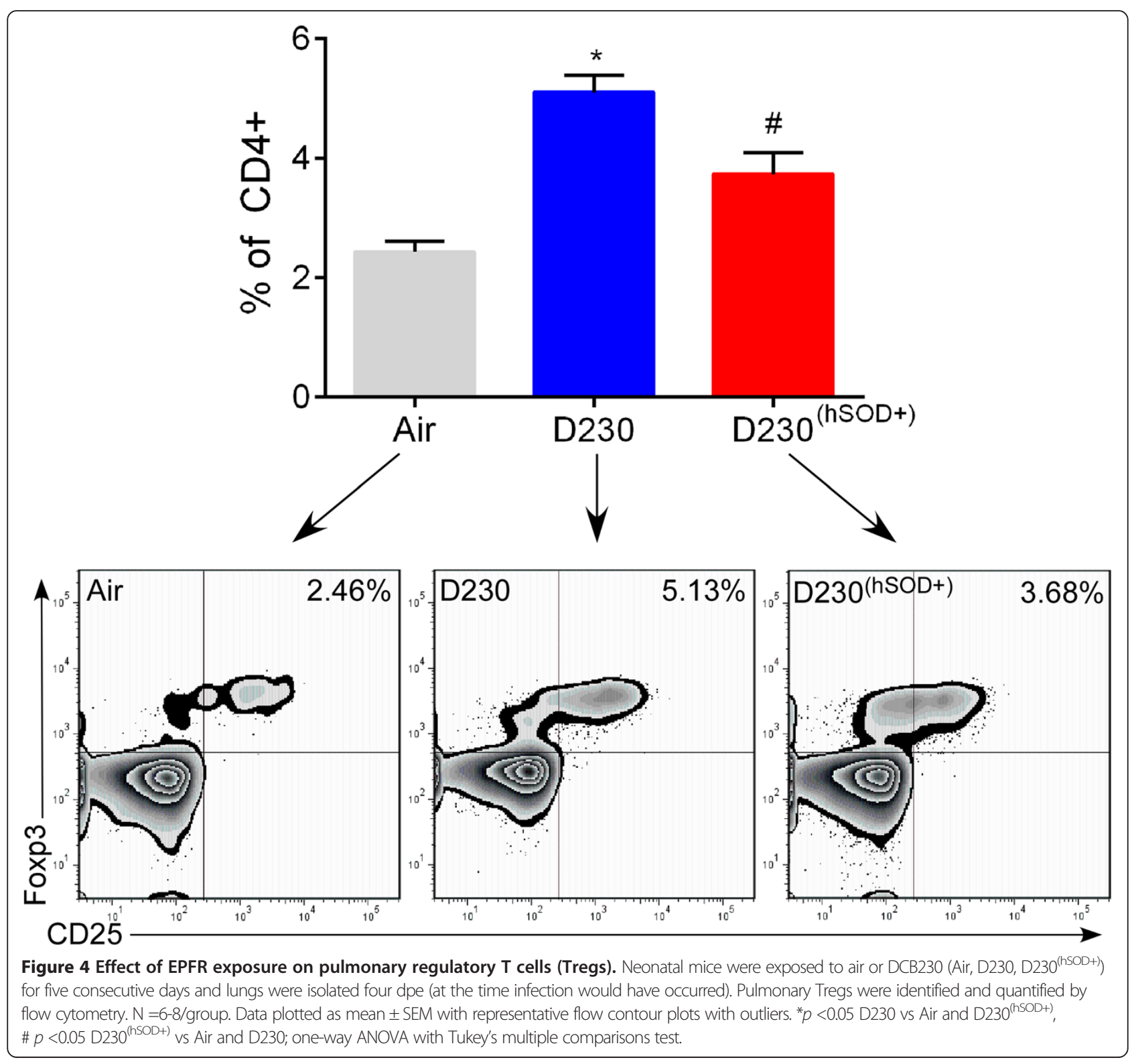


and Th1 cells compared to D230F (Additional file 4: Figure S4).

\section{Oxidative stress following exposure to EPFRs induces an increase in regulatory $T$ cells in the lungs}

Our previous data demonstrated that acute exposure to combustion derived PM induced a population of immunosuppressive T cells - Tregs [15]. However, from those studies it was unclear if this was due to the presence of EPFRs in the PM samples or due to epithelial injury. To determine if exposure to EPFRs induced a Treg response, we isolated lung cells at four dpe (i.e., the time of maximal epithelial injury and at the time influenza infection would have occurred in our model; Figure 1A) and quantified Tregs using flow cytometry. We observed significant increases in the percentage of Tregs in the lungs of neonates exposed to DCB230 compared to Air (Figure 4). The expression of hSOD2 resulted in a significant decrease in the percentage of pulmonary Tregs compared to D230; however, hSOD2 expression was not able to return Tregs numbers to homeostatic baseline levels.

\section{Discussion}

The association between PM exposure and enhanced respiratory viral disease severity in children is well documented. Studies that have investigated this phenomenon in adult animals have given important insights to this association [22], but it is also important to understand these responses in vulnerable subpopulations such as infants. Our studies, along with others, show that response (immunological or cellular) to agents (infectious and noninfectious) is markedly different between adults and neonates [14,15,23-28]. Our lab has already identified that pre-natal EPFR exposure increases the risk of asthma development in offspring [29]. Since infants are still undergoing dramatic pulmonary and immunological development, insults during this critical time period may incur additional and long term health consequences. Furthermore, the discovery of EPFRs present in real world PM from almost all combustion sources suggests its role in the adverse health effects associated with PM exposure [30]. This manuscript presents a novel model to study the effects of PM exposure and influenza infection in neonates, as well as, explores the effects of airborne EPFR exposure in neonates on influenza severity. Our neonatal model recapitulated what has been observed in epidemiological studies - enhanced severity of respiratory tract viral infection following exposure to elevated levels of PM [19-21,31]. In addition, our data demonstrated that exposure to PM containing EPFRs induced oxidative stress in neonates. This oxidative stress could be attenuated by increasing the antioxidant capacity of the host. Furthermore, our data revealed specifically that EPFR exposure, and not just PM exposure (DCB50), exacerbated morbidity due to influenza as evidenced by reductions in weight gain and higher pulmonary viral loads at both four and eight dpi (peak viral titer and viral clearance, respectively). More strikingly, EPFR exposure induced premature and decreased survival following influenza infection. Increased antioxidant capacity of the host was able to attenuate EPFR-enhanced morbidity and enhance survival following influenza infection.

Since the adaptive immune response is paramount in the host defense against pathogens, we focused our studies on the subset of CD8+ and CD4+ T cells instrumental in controlling and clearing intracellular pathogens such as influenza [32,33]. We discovered that both the subset of CD8+ and CD4+ T cells that release IFN $(\mathrm{Tc} 1$ and Th1, respectively) were significantly decreased following EPFR exposure in neonates. Due to the observed immunosuppressed phenotype in EPFR exposed and influenza infected neonates, we investigated the role of Tregs in EPFR exposed non-infected neonates because of their known role in immunosuppression. Our previously published data showed increased Treg numbers that coincided with increased epithelial injury even in the absence of influenza infection indicating that Tregs are not increased with increased viral load but instead they are induced by epithelial injury [34]. To determine whether Tregs induced solely due to EPFR exposure are responsible for the immunosuppressive environment in the lungs, we analyzed Treg responses prior to influenza infection (four dpe), at the time of maximum lung injury. Our data revealed that EPFR exposure alone significantly increased the percentage of Tregs in the lungs of neonates. This increase in Tregs could potentially explain the dampened Tc1 and Th1 effector response in influenza infected neonates exposed to EPFRs. In fact, in our prior study, we observed a similar phenomenon - early exposure to EPFRs resulted in Treg expansion and suppression of $\mathrm{T}$ cell responses to allergen sensitization and challenge (i.e., mouse asthma model) [15]. Importantly, Treg expansion in this model, although transient, appeared to create a window of vulnerability to infection. Indeed, our data presented here support our prior findings and clearly demonstrate that neonatal mice exposed to EPFRs are more vulnerable to influenza infection and exhibit enhanced morbidity and decreased percentage survival following infection. Cumulatively, our data suggest that this window of vulnerability resulting from exposure to EPFR-containing PM will dampen the ability to mount protective adaptive immune responses to any pathogen and would ultimately lead to inability to clear the pathogen and more severe disease, as was observed here.

Our data also demonstrated that exposure to PM containing EPFRs induced both oxidative damage (as evidenced by increased levels of 8-IP) and redox imbalances (as evidenced by decreased GSH/GSSG ratios) in the lungs. Together these markers suggest that the lung was in 
a state of oxidative stress prior to infection. To determine the association between oxidative stress in the enhanced influenza severity following EPFR exposure, neonatal mice were exposed to non-EPFR containing PM (DCB50) and we included neonates with a higher antioxidant capacity throughout our studies $\left(\mathrm{hSOD}^{+}\right)$. D50 and D230 ${ }^{(\mathrm{hSOD}+)}$ neonates exhibited lower pulmonary oxidative stress. Even more exciting, our data showed that PM exposure in the absence of an EPFR, or in the presence of an EPFR but within a host with enhanced antioxidant capacity, resulted in significantly less morbidity and enhanced survival in neonatal mice following infection with influenza. The improvement in morbidity and survival was associated with an improvement in the adaptive immune response to influenza (Tc1 and Th1) and a reduction in immune regulating $\mathrm{T}$ cells (Tregs). Taken together, our data identified pulmonary oxidative stress as a key component in the pathogenesis of PM enhanced influenza disease severity.

A related study by another laboratory looking at influenza viral loads in adult mice after exposure to diesel exhaust observed similar increases in viral load [22]. Although both studies identified increased severity of influenza mediated disease, only our study demonstrated a significant benefit associated with elevated antioxidant capacity (i.e., decreased pulmonary viral load and improved survival). In addition, we were able to show that oxidative stress induced by EPFR exposure resulted in immunosuppression, which could be alleviated by increasing the antioxidant capacity during the exposure resulting in enhanced protective immune responses. These incongruences could be explained by a myriad of differences between our studies including age (adult vs. neonate), strain of the mice (BALB/c vs. C57Bl/6), timing of the exposure to diesel exhaust or EPFR containing PM (post-infection vs. before and during infection), and the type, dose, and route of antioxidant utilized ( $\mathrm{N}$-acetylcysteine vs. SOD2).

Other studies have attempted to explain how exposure to PM exacerbates respiratory viral infections in neonates as a model to understand similar findings in human infants. Some have speculated that PM facilitates the spread of influenza by acting as condensation nuclei for viral droplets [35]. Other studies report enhanced viral attachment due to exposure to diesel exhaust [36], another source of EPFRs. The role of oxidative stress has also been studied and investigators report that oxidative stress inhibits GSH [37] which can lead to enhanced virus replication [38]. Oxidative stress can also inhibit mucus antiproteases [39] that regulate the trypsin like serine proteases which cleaves influenza hemagluttinin, effectively enhancing its ability to spread and more readily infect epithelial cells $[40,41]$. Our study proposes an additional mechanism for this phenomenon. First, our data suggest that exposure to PM containing EPFRs, which is found in most combustion derived PM, is responsible for the exacerbation of influenza in neonates. In addition, EPFR exposure resulted in enhanced pulmonary oxidative stress that led to increased pulmonary Tregs prior to infection. Our previous data suggested that the increased Tregs in the lungs results in an immunosuppressive environment that subsides within a week if exposure to EPFRs is discontinued [15]. We believe that the persistence of this immunosuppressive environment during and throughout the infection dampens the development of a protective adaptive immune response to influenza infection. Ultimately this is responsible for the increased morbidity and decreased survival observed. The effects of EPFR exposure were subsequently attenuated when antioxidant capacity was increased.

\section{Conclusions}

We demonstrate that EPFRs associated with combustion derived PM are important in enhancing severity and mortality following respiratory tract viral infections. Our data demonstrate the benefit of antioxidant supplementation in reversing the enhanced disease due to influenza infection in the context of EPFR-containing PM exposure. The antioxidant data further suggest that it may be clinically beneficial to identify at-risk infants (i.e., those who reside in high traffic areas, reside in homes with smokers, live in homes where biomass fuels are used, etc.), so that supplementation with antioxidants prior to influenza season may be employed to reduce disease burden. It is important to note that the results from this study only suggest the benefits of prophylactic usage of antioxidants prior to/ concurrent with exposure to EPFRs. It is worthwhile to study the effectiveness of antioxidants as a therapeutic agent, post-exposure and potentially post-infection. $\mathrm{Fi}$ nally, it would be interesting to investigate the role of EPFR exposure and secondary or concurrent bacterial/ viral infection in neonates as mortality incidences are astronomically high in these patients. In summary, this study illustrates a unique neonatal model to further investigate the mechanism by which air pollution modulates respiratory viral illnesses and disease. This model can easily be extrapolated to other non-viral or non-pathogenic insults that infants commonly encounter.

\section{Methods}

\section{Combustion derived PM}

PM with a mean aerodynamic diameter of $0.2 \mu \mathrm{m}$ was generated and characterized by our colleague (Dr. Slawo Lomnicki, LSU) as previously described $[17,18]$. Briefly, combustion derived PM containing a chemisorbed EPFR (DCB230) or lacking an EPFR (DCB50) was suspended in $25 \mathrm{~mL}$ of irrigation saline containing $0.02 \%$ Tween- 80 resulting in a final concentration of $0.2 \mathrm{mg} / \mathrm{mL}$. The particles were monodispersed via probe sonication immediately prior to use. 


\section{Animals}

C57BL/6NHsd breeders were purchased from Harlan (Indianapolis, IN). Human SOD2 transgenic mice were generously provided by Nils-Goran Larsson of the Max Planck Institute for Biology of Ageing (Cologne, Germany). All animals were housed in ventilated cages in a specific pathogen free environment and time mated to maximize birth cohorts. Pups expressing the hSOD2 transgene $\left(\mathrm{hSOD}^{+}\right)$were generated by crossing heterozygous hSOD2/+ male mice to wildtype C57BL/6 (WT) females. The presence of the transgene was confirmed by PCR. Littermates not expressing hSOD2 (hSOD ${ }^{-}$) were used as controls. Separate cohorts of pups were used for independent experimental end points (oxidative stress or flow cytometry or viral load). Neonatal mice were not identified for sex; and therefore, mice of both sexes were used for all the experiments. All animal protocols were prepared in accordance with the Guide for the Care and Use of Laboratory Animals and approved by the LSUHSC and UTHSC Institutional Animal Care and Use Committee.

\section{Neonatal exposures}

Neonatal mice, defined as less than seven days of age, were exposed to air, DCB50, or DCB230. To mimic a human infant exposure, we relied on modeling data (MPPD v2.0) to produce an equivalent particle deposition per alveolus to that of an infant human being exposed over a $24 \mathrm{~h}$ time period to an average of $35 \mathrm{ug} /$ $\mathrm{m}^{3}$ of PM2.5. Thus the whole body exposure dose was set to $200 \mu \mathrm{g} / \mathrm{m}^{3}$. The parameters used in the algorithm and how the air flow in the chamber were calculated and monitored were described previously [15]. A 3 day old mouse mimics a newborn human infant in regards to pulmonary development [13]. Hence, to mimic the exposure of a newborn human infant to particulate matter containing EPFRs, neonatal mice were exposed to EPFRs beginning on day 3 of age. Mice were placed in a chamber system in which particles were nebulized for 30 minutes/day for seven consecutive days reaching an average daily exposure concentration of $200 \mu \mathrm{g} / \mathrm{m}^{3}$. These exposure conditions were selected to mimic real-world exposure scenarios and were based on modeling calculations to achieve an equivalent alveolar deposition dose as in human infants as previously described [15].

\section{Neonatal influenza model}

Neonatal pups were infected four dpe i.n. with 1.25 $\mathrm{TCID}_{50}$ /neonate of mouse adapted human influenza A strain PR/8/34 H1N1 (Advanced Biotechnologies, MD) in $10 \mu \mathrm{L}$ of Dulbecco's phosphate-buffered saline (DPBS). Non-infected neonates were dosed with the same volume of DPBS. Weight gain and survival were assessed daily, immediately following infection, for the duration of the protocol (Figure 1).

\section{Markers of oxidative stress (8-isoprostanes, GSH/GSSG ratio)}

Lungs were excised after five days of exposure (at the time infection would have occurred) and immediately flash frozen in liquid nitrogen for analysis for 8-IP and GSH/ GSSG. Samples were saponified and purified as described previously [42] and 8-IP was quantified by ELISA (Cayman, MI) as per the manufacturer's protocol. GSH/GSSG was detected using HPLC electrochemical detection as previously described [16].

\section{Pulmonary viral load}

Lungs were excised and isolated at four dpi and eight dpi. Lungs were homogenized and viral titer was determined using the TCID 50 method of Spearman and Kärber $[43,44]$. Briefly, Madin-Darby canine kidney cells were seeded at a density of $1.5 \times 10^{4}$ cells/well on a 96 well flat bottom plate. Cells were subsequently inoculated with a 10 -fold dilution series of homogenized lungs. Cells were incubated at $37^{\circ} \mathrm{C}$ with $5 \% \mathrm{CO}_{2}$ for five days, observed daily for signs of cytopathic effect. Scoring for cytopathic effect was finalized on day five and $\mathrm{TCID}_{50}$ calculated.

\section{Flow cytometry and intracellular staining}

Pups were sacrificed and blood was gently flushed from the lungs by retrograde perfusion with isotonic saline. A single lung cell suspension was prepared as previously described $[15,25,45]$. Briefly, perfused lungs were isolated, collected in chilled Hank's balanced salt solution (HBSS), and mechanically dissociated using the Octodissociator (Miltenyi, Germany). Dissociated lungs were incubated and digested at $37^{\circ} \mathrm{C}$ with continuous shaking (200 rpm) for 30 minutes in HBSS supplemented with $1 \mathrm{mg} / \mathrm{mL}$ collagenase I (Invitrogen, NY), and $150 \mathrm{ng} / \mathrm{mL}$ DNase I (Sigma Aldrich, MO). Following incubation, lungs were dissociated a second time with the Octodissociator and remaining cell clumps dissociated by straining through a $40 \mu \mathrm{m}$ cell strainer (BD Biosciences, $\mathrm{CA}$ ). The resulting single cell lung suspension was further treated with RBC lysis buffer to remove residual red blood cells. Cells were stimulated for intracellular cytokine staining for five hours at $37^{\circ} \mathrm{C}$ in RPMI 1640 media containing 5\% heat-inactivated fetal bovine serum, $5 \mathrm{ng} / \mathrm{mL}$ phorbol 12-myristate 13-acetate, and $500 \mathrm{ng} / \mathrm{mL}$ ionomycin (Sigma-Aldrich) in the presence of a protein transport inhibitor (GolgiPlug, BD Biosciences). For $\mathrm{T}$ cell staining, the following antibodies were used: eFluor450-CD3 (17A2; eBiosciences, CA), FITCCD8 (53-6.7; eBiosciences), PerCP-CD4 (RM4-5; BioLegend, CA), and PE-IFNY (XMG1.2; eBiosciences). For regulatory $\mathrm{T}$ cell staining, the following antibodies were used: eFluor450-CD3 (17A2; eBiosciences), PerCP-CD4 (RM4-5; BioLegend), PE-CD25 (PC61.5; eBiosciences), FITC-Foxp3 (FJK-16s; eBiosciences). Dead cells were excluded using a fixable live/dead marker eFluor 780 
(eBiosciences). Stained cells were analyzed with a FACS Canto II (BD Biosciences). Flow data were analyzed and plotted using FlowJo Software v.7.6.5 (Tree Star, OR).

\section{Statistical analysis}

All results are expressed as mean \pm SEM and were analyzed using GraphPad Prism 6 (GraphPad Software Inc., Version 6.03, CA). One way analysis of variance (ANOVA) with Tukey's multiple comparisons tests, multiple $t$ tests with Holm-Sidak correction for multiple comparisons, and unpaired $t$ test were used to determine differences between groups. Differences between survival curves were analyzed using Gehan-Breslow Wilcoxon test with Bonferroni correction for multiple comparisons. $P$ values less than 0.05 were considered statistically significant, except where noted (survival curve analysis).

\section{Additional files}

Additional file 1: Figure S1. EPFR exposure induces oxidative stress in hSOD2 negative (hSOD2) littermate control neonates. WT or hSODneonates were exposed to air or DCB230 (Air, D230, Air ${ }^{\text {(hSOD-) }}$, D230 ${ }^{(\text {hSOD-) }}$ ). Pulmonary oxidative stress was determined by assessing levels of (A) 8-IP and (B) GSH/GSSG after five days of exposure. N =4-10/group. Data from mice exposed to DCB230 was normalized to respective Air controls, data plotted as mean \pm SEM. $p>0.05$; Unpaired t test.

Additional file 2: Figure S2. Effect of EPFR exposure on influenza morbidity in hSOD2 littermate control neonates. Neonates were exposed to Air or DCB230 and infected i.n. with influenza (AirF, D230F) at 1.25 $\mathrm{TCID}_{50}$ /neonate. (A) Pulmonary peak viral titer assessed at four dpi. $N=5-22 /$ group. (B) Pulmonary viral clearance assessed at eight $d p i$. $\mathrm{N}=6-18$ /group. Data plotted as mean \pm SEM. $p<0.05$ (brackets); one-way analysis of variance (ANOVA) with Tukey's multiple comparisons test.

Additional file 3: Figure S3. Peak pulmonary viral load assessed in WT (AirF), hSOD2 transgenic ( $h+$ AirF), and transgenic littermates lacking hSOD2 (h-AirF) neonates at four days post-infection. Neonates were infected i.n. with influenza at $1.25 \mathrm{TCID}_{50} /$ neonate and peak pulmonary viral load was assessed at four dpi. N =6-7/group. Dotted line indicates limit of detection $\left(35 \mathrm{TCID}_{50} / \mathrm{mL}\right.$ ) of assay. Data plotted as mean $\pm \mathrm{SEM}$. p >0.05; Dunn's multiple comparisons test.

Additional file 4: Figure S4. Effect of EPFR exposure on pulmonary adaptive $T$ cell responses (TC1 and Th1) after infection to influenza in neonatal mice. Mice were exposed to air, DCB50, or DCB230 and infected i.n. with influenza (AirF, D50F, D230F, D230F ${ }^{(\mathrm{hSOD+})}$ ) or sham infected with DPBS (Air) at four dpe. Total lung effector $T$ cell profiles were determined by flow cytometry at six dpi. (A) Total number of CD8+ cells expressing IFNy (TC1) with representative flow contour plots demonstrating outliers. $\mathrm{N}=3-12$ /group. (B) Total number of CD4+ cells expressing IFNy (Th1) with representative flow contour plots demonstrating outliers. $N=3-12$ / group. Data plotted as mean \pm SEM. ${ }^{*} p<0.05$ D230F vs AirF, D50F, and D230F ${ }^{(\mathrm{hSOD}+)}$; multiple t tests with Holm-Sidak correction for multiple comparisons.

\section{Abbreviations}

EPFR: Environmentally persistent free radicals; DCB230: EPFR containing PM; SOD2: Superoxide dismutase $2, \mathrm{hSOD}^{+}$, transgenic mouse expressing human SOD2; DCB50: PM lacking EPFR; 8-IP: 8-isoprostane; GSH/GSSG: Glutathione/ glutathione disulfide; hSOD: Littermate not expressing human SOD2; dpi: Day post-infection; i.n.: Intranasal; TC1: CD8+ T cell expressing IFNy; Th1: CD4+ T cell expressing IFNy; Tregs: Regulatory T cells; DPBS: Dulbecco's phosphate-buffered saline; HBSS: Hank's balanced salt solution;

SEM: Standard error of means; ANOVA: Analysis of variance.

\section{Competing interests}

The authors declare that they have no competing interests.

\section{Authors' contributions}

GIL and SAC conceived and designed the study, and drafted the manuscript. JS and DY assisted in the design of the study and flow cytometry experiments. BS assisted with flow cytometry experiments and genotyping. SJ assisted in exposure and flow cytometry. WH and TRD performed 8-IP ELISA and GSH/GSSG HPLC experiments. All authors have read and approved the manuscript.

\section{Authors' information}

GIL is an MD/PhD candidate interested in identifying novel therapies and observations tested in animal models and applying their usage clinically. SAC is interested in the neonatal and environmental origins of pulmonary disease.

\section{Acknowledgements}

This work was supported by NIEHS grants to SAC (R01ES015050 and P42ES013648) and NIAID grant to SAC (R01Al090059) and Gandolini Research Fellowship from LSUHSC - New Orleans to GIL.

\section{Author details}

'Department of Pharmacology and Experimental Therapeutics, Louisiana State University Health Sciences Center, New Orleans, Louisiana 70112, USA. ${ }^{2}$ Department of Pediatrics, University of Tennessee Health Sciences Center, Memphis, Tennessee 38103, USA. ${ }^{3}$ Children's Foundation Research Institute, Le Bonheur Children's Hospital, Memphis, Tennessee, USA. ${ }^{4}$ Department of Pharmacology, Toxicology and Neuroscience, Louisiana State University Health Sciences Center, Shreveport, Louisiana 71103, USA.

Received: 22 April 2014 Accepted: 8 October 2014

Published online: 30 October 2014

\section{References}

1. Pope CA 3rd, Burnett RT, Thun MJ, Calle EE, Krewski D, Ito K, Thurston GD: Lung cancer, cardiopulmonary mortality, and long-term exposure to fine particulate air pollution. JAMA 2002, 287:1132-1141.

2. Kumar N, Liang D, Comellas A, Chu AD, Abrams T: Satellite-based PM concentrations and their application to COPD in Cleveland, OH. J Expo Sci Environ Epidemiol 2013, 23:637-646.

3. Balmes JR, Cisternas M, Quinlan PJ, Trupin L, Lurmann FW, Katz PP, Blanc PD: Annual average ambient particulate matter exposure estimates, measured home particulate matter, and hair nicotine are associated with respiratory outcomes in adults with asthma. Environ Res 2014, 129:1-10.

4. Dockery DW: Health effects of particulate air pollution. Ann Epidemiol 2009, 19:257-263.

5. Valavanidis A, Fiotakis K, Vlachogianni T: Airborne particulate matter and human health: toxicological assessment and importance of size and composition of particles for oxidative damage and carcinogenic mechanisms. J Environ Sci Health C Environ Carcinog Ecotoxicol Rev 2008, 26:339-362.

6. Vandini S, Corvaglia L, Alessandroni R, Aquilano G, Marsico C, Spinelli M, Lanari M, Faldella G: Respiratory syncytial virus infection in infants and correlation with meteorological factors and air pollutants. Ital J Pediatr 2013, 39:1

7. Fukuda K, Hider PN, Epton MJ, Jennings LC, Kingham SP: Including viral infection data supports an association between particulate pollution and respiratory admissions. Aust N Z J Public Health 2011, 35:163-169.

8. Saravia J, Lee GI, Lomnicki S, Dellinger B, Cormier SA: Particulate matter containing environmentally persistent free radicals and adverse infant respiratory health effects: a review. J Biochem Mol Toxicol 2013, 27:56-68.

9. Dellinger B, Pryor WA, Cueto R, Squadrito GL, Hegde V, Deutsch WA: Role of free radicals in the toxicity of airborne fine particulate matter. Chem Res Toxicol 2001, 14:1371-1377.

10. Braun-Fahrlander C, Ackermann-Liebrich U, Schwartz J, Gnehm HP, Rutishauser M, Wanner HU: Air pollution and respiratory symptoms in preschool children. Am Rev Respir Dis 1992, 145:42-47.

11. Ko FW, Tam W, Wong TW, Lai CK, Wong GW, Leung TF, Ng SS, Hui DS: Effects of air pollution on asthma hospitalization rates in different age groups in Hong Kong. Clin Exp Allergy 2007, 37:1312-1319. 
12. Arriola CS, Brammer L, Epperson S, Blanton L, Kniss K, Mustaquim D, Steffens C, Dhara R, Leon M, Perez A, Chaves SS, Katz J, Wallis T, Villanueva J, Xu X, Abd Elal Al, Gubareva L, Cox N, Finelli L, Bresee J, Jhung M: Update: influenza activity - United States, September 29, 2013-February 8, 2014 MMWR Morb Mortal Wkly Rep 2014, 63:148-154.

13. Amy RW, Bowes D, Burri PH, Haines J, Thurlbeck WM: Postnatal growth of the mouse lung. J Anat 1977, 124:131-151.

14. Cormier SA, You D, Honnegowda S: The use of a neonatal mouse model to study respiratory syncytial virus infections. Expert Rev Anti Infect Ther 2010, 8:1371-1380.

15. Saravia J, You D, Thevenot P, Lee Gl, Shrestha B, Lomnicki S, Cormier SA: Early-life exposure to combustion-derived particulate matter causes pulmonary immunosuppression. Mucosal immunology 2014, 7:694-704.

16. Thevenot PT, Saravia J, Jin N, Giaimo JD, Chustz RE, Mahne S, Kelley MA, Hebert W, Dellinger B, Dugas TR, Demayo FJ, Cormier SA: Radical-containing ultrafine particulate matter initiates epithelial-to-mesenchymal transitions in airway epithelial cells. Am J Respir Cell Mol Biol 2013, 48:188-197.

17. Lomnicki S, Truong H, Vejerano E, Dellinger B: Copper oxide-based model of persistent free radical formation on combustion-derived particulate matter. Environ Sci Technol 2008, 42:4982-4988.

18. Balakrishna S, Saravia J, Thevenot P, Ahlert T, Lominiki S, Dellinger B, Cormier SA: Environmentally persistent free radicals induce airway hyperresponsiveness in neonatal rat lungs. Part Fibre Toxicol 2011, 8:11.

19. Smith KR, Samet JM, Romieu I, Bruce N: Indoor air pollution in developing countries and acute lower respiratory infections in children. Thorax 2000, 55:518-532.

20. Pandey MR, Regmi HN, Neupane RP, Gautam A, Bhandari DP: Domestic smoke pollution and respiratory function in rural Nepal. Tokai J Exp Clin Med 1985, 10:471-481.

21. Schwartz J, Spix C, Wichmann HE, Malin E: Air pollution and acute respiratory illness in five German communities. Environ Res 1991, 56:1-14.

22. Gowdy KM, Krantz QT, King C, Boykin E, Jaspers I, Linak WP, Gilmour MI: Role of oxidative stress on diesel-enhanced influenza infection in mice. Part Fibre Toxicol 2010, 7:34.

23. You D, Marr N, Saravia J, Shrestha B, Lee Gl, Turvey SE, Brombacher F, Herbert DR, Cormier SA: IL-4Ralpha on CD4+ T cells plays a pathogenic role in respiratory syncytial virus reinfection in mice infected initially as neonates. J Leukoc Biol 2013, 93:933-942.

24. Chan JK, Charrier JG, Kodani SD, Vogel CF, Kado SY, Anderson DS, Anastasio C, Van Winkle LS: Combustion-derived flame generated ultrafine soot generates reactive oxygen species and activates Nrf2 antioxidants differently in neonatal and adult rat lungs. Part Fibre Toxicol 2013, 10:34.

25. You D, Ripple M, Balakrishna S, Troxclair D, Sandquist D, Ding L, Ahlert TA, Cormier $\mathrm{SA}$ : Inchoate CD8+ T cell responses in neonatal mice permit influenza-induced persistent pulmonary dysfunction. J Immunol 2008, 181:3486-3494.

26. You D, Becnel D, Wang K, Ripple M, Daly M, Cormier SA: Exposure of neonates to respiratory syncytial virus is critical in determining subsequent airway response in adults. Respir Res 2006, 7:107.

27. Wang P, Thevenot P, Saravia J, Ahlert T, Cormier SA: Radical-containing particles activate dendritic cells and enhance Th17 inflammation in a mouse model of asthma. Am J Respir Cell Mol Biol 2011, 45:977-983.

28. Thevenot P, Saravia J, Giaimo J, Happel Kl, Dugas TR, Cormier SA: Chronic alcohol induces M2 polarization enhancing pulmonary disease caused by exposure to particulate air pollution. Alcohol Clin Exp Res 2013, 37:1910-1919.

29. Wang P, You D, Saravia J, Shen H, Cormier S: Maternal exposure to combustion generated PM inhibits pulmonary Th1 maturation and concomitantly enhances postnatal asthma development in offspring. Part Fibre Toxicol 2013, 10:29.

30. Steenhof M, Gosens I, Strak M, Godri KJ, Hoek G, Cassee FR, Mudway IS, Kelly FJ, Harrison RM, Lebret E, Brunekreef B, Janssen NA, Pieters RH: In vitro toxicity of particulate matter (PM) collected at different sites in the Netherlands is associated with PM composition, size fraction and oxidative potential-the RAPTES project. Part Fibre Toxicol 2011, 8:26.

31. Xu Z, Hu W, Williams G, Clements AC, Kan H, Tong S: Air pollution, temperature and pediatric influenza in Brisbane, Australia. Environ Int 2013, 59:384-388.

32. Stambas J, Guillonneau C, Kedzierska K, Mintern JD, Doherty PC, La Gruta NL: Killer T cells in influenza. Pharmacol Ther 2008, 120:186-196.

33. Sun J, Madan R, Karp CL, Braciale TJ: Effector T cells control lung inflammation during acute influenza virus infection by producing IL-10. Nat Med 2009, 15:277-284.
34. Josefowicz SZ, Lu LF, Rudensky AY: Regulatory T cells: mechanisms of differentiation and function. Annu Rev Immunol 2012, 30:531-564.

35. Hammond GW, Raddatz RL, Gelskey DE: Impact of atmospheric dispersion and transport of viral aerosols on the epidemiology of influenza. Rev Infect Dis 1989, 11:494-497.

36. Jaspers I, Ciencewicki JM, Zhang W, Brighton LE, Carson JL, Beck MA Madden MC: Diesel exhaust enhances influenza virus infections in respiratory epithelial cells. Toxicol Sci 2005, 85:990-1002.

37. Balakrishna S, Lomnicki S, McAvey KM, Cole RB, Dellinger B, Cormier SA: Environmentally persistent free radicals amplify ultrafine particle mediated cellular oxidative stress and cytotoxicity. Part Fibre Toxicol 2009, $6: 11$.

38. Nencioni L, luvara A, Aquilano K, Ciriolo MR, Cozzolino F, Rotilio G, Garaci E, Palamara AT: Influenza A virus replication is dependent on an antioxidant pathway that involves GSH and Bcl-2. The FASEB Journal 2003, 17(6):758-760.

39. Cavarra E, Lucattelli M, Gambelli F, Bartalesi B, Fineschi S, Szarka A, Giannerini F, Martorana PA, Lungarella G: Human SLPI inactivation after cigarette smoke exposure in a new in vivo model of pulmonary oxidative stress. Am J Physiol Lung Cell and Mol Physiol 2001, 281(2):L412-L417.

40. Kido H, Beppu Y, Imamura Y, Chen Y, Murakami M, Oba K, Towatari T: The human mucus protease inhibitor and its mutants are novel defensive compounds against infection with influenza $A$ and Sendai viruses. Peptide Science 1999, 51:79-86.

41. Kido H, Yokogoshi Y, Sakai K, Tashiro M, Kishino Y, Fukutomi A, Katunuma N Isolation and characterization of a novel trypsin-like protease found in rat bronchiolar epithelial Clara cells. A possible activator of the viral fusion glycoprotein. J Biological Chemistry 1992, 267:13573-13579.

42. Fahmy B, Ding L, You D, Lomnicki S, Dellinger B, Cormier SA: In vitro and in vivo assessment of pulmonary risk associated with exposure to combustion generated fine particles. Environmental toxicol and pharmacol 2010, 29:173-182

43. Kärber G: Beitrag zur kollektiven Behandlung pharmakologischer Reihenversuche. Archiv f experiment Pathol u Pharmakol 1931, 162:480-483.

44. Spearman C: The method of 'right and wrong cases' ('constant stimuli') without gauss's formulae. British J Psychology, 1904-1920 1908, 2:227-242.

45. Ormerod MG: Preparing suspensions of single cells. In Flow cytometry: A practical approach. 3rd edition. Oxford: University Press; 2000:35-46.

\section{doi:10.1186/s12989-014-0057-1}

Cite this article as: Lee et al:: Exposure to combustion generated environmentally persistent free radicals enhances severity of influenza virus infection. Particle and Fibre Toxicology 2014 11:57.

\section{Submit your next manuscript to BioMed Central and take full advantage of:}

- Convenient online submission

- Thorough peer review

- No space constraints or color figure charges

- Immediate publication on acceptance

- Inclusion in PubMed, CAS, Scopus and Google Scholar

- Research which is freely available for redistribution
C) Biomed Central 